\title{
Chlamydia pneumoniae y aterosclerosis: Mecanismos patogénicos
}

\author{
ORISON WOOLCOTT, LUIS SÁNCHEZ \\ Facultad de Medicina, Universidad Nacional Mayor de San Marcos.
}

\begin{abstract}
RESUMEN
$\mathrm{La}$ aterosclerosis es un proceso inflamatorio crónico en donde intervienen macrófagos, células musculares lisas y linfocitos T, además de un conjunto de mediadores químicos. En los últimos años, el hallazgo de Chlamydia pneumoniae en placas ateroscleróticas en diferentes regiones de nuestro cuerpo ha sugerido un rol etiológico de este microorganismo en el proceso de aterogénesis. Sin embargo, todavía es incierto si la C. pneumoniae es el agente causal de la aterosclerosis o si constituye un factor que precipita o favorece su progresión. Se ha hecho una revisión de artículos publicados hasta la actualidad, disponibles como texto completo vía internet o en las bibliotecas especializadas de Lima, acerca de la asociación entre C. pneumoniae y aterosclerosis, poniendo énfasis en el rol patogénico de esta bacteria.
\end{abstract}

Palabras clave: Aterosclerosis; Chlamydia pneumoniae; bacterias gramnegativas.

\section{Chlamydia pneumoniae AND ATHEROSCLEROSIS: PATHOGENIC WAYS SUMMARY}

Atherosclerosis is a chronic inflammatory process in which macrophages, smooth muscle cells, $\mathrm{T}$ lymphocytes, and several chemical mediators intervene. In recent years, the finding of Chlamydia pneumoniae in arterial atherosclerotic plaques has suggested an etiological role; however, whether C. pneumoniae causes atherosclerosis or precipitates or favors atheroesclerosis progression remains uncertain. We review studies published on-line or available in Lima specialized libraries regarding $C$. pneumoniae and atherosclerosis association, with special emphasis on the pathogenic role of this bacteria.

Key words: Atherosclerosis; Chlamydia pneumoniae; gram-negative bacteria.

En 1965, Grayston y colaboradores $\left({ }^{1}\right)$ aislaron por primera vez a la Chlamydia pneumoniae, tipificada como TW-183. Posteriormente, en 1985, se notificó una epidemia de neumonía causada por esta cepa $\left({ }^{2}\right)$. Un año después, se reportó el aislamiento de una cepa de Chlamydia, denominada AR-39 $\left({ }^{3}\right)$, a partir de la secreción faríngea de pacientes con infección respirato-

Correspondencia:

Dr. Orison Woolcott

Calle Alcalá 132, Mayorazgo

Lima 3, Perú

E-mail: orisonwc@terramail.com.pe ria. Estas dos cepas de $C$. pneumoniae tienen gran similitud morfológica con la $C$. psittaci, agente causal de la psitacosis. Por esta razón, inicialmente fueron consideradas como cepas de C. psittaci $\left({ }^{4}\right)$. Actualmente se conoce cuatro especies de Chlamydia: trachomatis, psittaci, pneumoniae y pecorum $\left(^{5}\right)$.

Las Chlamydiae son bacterias gram-negativas que se caracterizan por presentar dos antígenos mayores, la proteína de choque por calor 60 (HSP 60) y la proteína mayor de membrana externa $\left({ }^{6}\right)$. Poseen un ciclo de vida bajo dos formas: en el extracelular, la Chlamydia tiene capacidad infectante, pero es metabólicamente 
inactiva; a esta forma se le llama cuerpo elemental; una vez fagocitado por el macrófago, adopta una forma con capacidad de replicación, denominado cuerpo reticular. Las Chlamydia son de vida intracelular obligada, ya que carecen de enzimas de la cadena de electrones para su propio metabolismo $\left({ }^{7}\right)$.

Existen diversos procedimientos para el diagnóstico de infección por Chlamydia, entre ellos el aislamiento de la bacteria, el método de inmunofluorescencia $\left({ }^{8}\right)$ y el método de tinción por inmunohistoquímica $\left({ }^{9}\right)$. Desde la década pasada se está empleando métodos más costosos como la reacción en cadena de la polimerasa $\left({ }^{10}\right)$ y la microscopia electrónica $\left({ }^{11}\right)$.

Para el diagnóstico serológico de $C$. pneumoniae sólo se considera los anticuerpos IgG e IgM. El diagnóstico de infección aguda se determina por un título de $\operatorname{IgM} \geq 1: 16$ ó un incremento de 4 veces el valor de IgG. El diagnóstico de infección antigua se hace con un título de $\operatorname{IgG} \geq 1: 16\left({ }^{12}\right)$.

\section{Estudios epidemiológicos}

Una de las enfermedades que causa gran morbilidad y mortalidad en todo el mundo, principalmente en los países desarrollados, es el infarto miocárdico agudo (IMA) y, en la mayoría de casos, el IMA está relacionado con la aterosclerosis $\left({ }^{13,14}\right)$.

En diversas investigaciones epidemiológicas, se ha encontrado una asociación entre Chlamydia pneumoniae y aterosclerosis $\left({ }^{15-19}\right)$. Los Centros para el Control y Prevención de Enfermedades (CDC) han incluido esta asociación en el contexto de enfermedades emergentes del nuevo siglo $\left({ }^{20}\right)$, lo que traduce la importancia que representan los hallazgos que se tiene hasta la fecha con relación a aterosclerosis y $C$. pneumoniae.

Los estudios epidemiológicos señalan que existe una mayor prevalencia de infección por C. pneumoniae en el sexo masculino ( $80 \%$ ) que en el sexo femenino $(70 \%)$, en la población adul- ta $\left({ }^{21}\right)$. Un elemento que favorece el crecimiento de las bacterias gram-negativas es el hierro, y se conoce que las arterias ateroscleróticas contienen más hierro que las arterias sin aterosclerosis. Además, se sabe que un varón adulto posee más hierro que una mujer adulta. Entonces, desde ese punto de vista, los varones serían más susceptibles a la persistencia de una infección por C. pneumoniae ${ }^{(22)}$.

Existe la hipótesis que dentro de la placa ateromatosa la $C$. pneumoniae no ejerce algún efecto patogénico y sólo sea un "espectador" $\left.{ }^{18}\right)$, y que los macrófagos puedan fagocitar a la C. pneumoniae en los pulmones y migrar hacia zonas ateroscleróticas, donde podría residir sin causar daño ( ${ }^{23}$ ). En numerosos estudios se observa que la $C$. pneumoniae se encuentra en la placa aterosclerótica de diferentes regiones de nuestro cuerpo, como por ejemplo en las arterias coronarias $\left({ }^{24-27}\right)$, en el cayado de la aorta ${ }^{(28)}$, en la arteria carótida $\left({ }^{29}\right) \mathrm{y}$, raramente, en arterias sin aterosclerosis $\left({ }^{9,30}\right)$. Asimismo, varios investigadores han reportado títulos elevados de anticuerpos contra $C$. pneumoniae en pacientes con cardiopatía coronaria $\left({ }^{31-34}\right)$, con aterosclerosis asintomática de la carótida $\left({ }^{35}\right)$ y en pacientes con insuficiencia crónica renal $\left({ }^{36}\right)$. También se ha encontrado $C$. pneumoniae en órganos como el pulmón, el hígado, bazo, médula ósea y ganglios linfáticos $\left({ }^{37}\right)$.

Debido a la existencia de diversas pruebas de laboratorio para determinar la presencia de C. pneumoniae -microinmunofluorescencia $\left({ }^{38}\right)$, inmunohistoquímica $\left({ }^{11}\right)$, cultivo $\left({ }^{39}\right)$, microscopia electrónica $\left({ }^{24,25}\right)$, reacción en cadena de polimerasa $\left({ }^{10,27,40-42}\right)$ e hibridización in situ $\left({ }^{25}\right)$ - y la ausencia de métodos estandarizados, hay mucha discrepancia entre los resultados encontrados por diferentes grupos de investigadores. Así, mediante el PCR se ha detectado $C$. pneumoniae en lesiones ateroscleróticas entre $30 \%$ y $89 \%\left({ }^{43,44}\right)$, mientras que por el método de inmunohistoquímica las cifras son menores de $50 \%\left({ }^{11,18,43,45}\right)$. Contrariamente, en algunos estudios no se encontró asociación entre $C$. pneumoniae y aterosclerosis 
mediante inmunofluorescencia $\left({ }^{40,46-50}\right)$. Frente a estos resultados variables, la CDC ha publicado una serie de lineamientos para estandarizar estas pruebas y los criterios diagnósticos del microorganismo. Para la detección de $C$. pneumoniae en suero, sólo se recomienda el uso de microinmunofluorescencia. El diagnóstico definitivo de un cultivo positivo requiere la propagación del cultivo aislado o la confirmación por PCR $\left({ }^{51}\right)$. Asimismo, para evitar falsos positivos con el uso de inmunohistoquí-mica es necesario un adecuado entrenamiento, para distinguir artefactos que captan la tinción y dan el aspecto de ser partículas de Chlamydia.

\section{Rol de la Chlamydia pneumoniae en la patogenia de la aterosclerosis}

La hipótesis de la injuria al endotelio formulada por Ross y colaboradores $\left({ }^{52}\right)$, en 1973 , revolucionó el concepto de la patogénesis de la aterosclerosis. La aterosclerosis es principalmente un proceso inflamatorio crónico, en el que están implicados macrófagos, células musculares lisas y linfocitos $\mathrm{T}$, además de un conjunto de mediadores, como el factor de crecimiento, citoquinas -tales como las interleuquinas 1,2 y 6 , el factor de necrosis tumoral $\alpha$, el interferón $\gamma$-, lipoproteínas de baja densidad (LDL), moléculas de adhesión a células vasculares $\left({ }^{14,53,54}\right)$, entre otras.

La presencia de C. pneumoniae en arterias ateroscleróticas sugiere algún rol etiológico de este microorganismo en el proceso de la aterogénesis. In vitro se ha demostrado que la C. pneumoniae puede infectar, sobrevivir y multiplicarse dentro de macrófagos derivados de monocitos, células musculares lisas de los vasos, células endoteliales $\left({ }^{55,56}\right)$ y adipocitos $\left({ }^{28}\right)$, lo que hace que las paredes de las arterias sean un hábitat "confortable" para estas bacterias.

Se postula que una re-infección por $C$. pneumoniae podría determinar el inicio de un IMA. Se ha demostrado una alta prevalencia de síntomas respiratorios semanas previas a la ocu- rrencia de un infarto $\left({ }^{57}\right)$. Los mecanismos inmunológicos de este proceso todavía no son muy claros, aunque se ha encontrado que la $C$. pneumoniae es capaz de inducir -en células endoteliales y musculares lisas vasculares- la activación de un conjunto de mediadores responsables de la respuesta inflamatoria, como la interleuquina 6 , así también moléculas procoagulantes, como el inhibidor-activador del plasminógeno 1, el factor tisular y el factor nuclear- $\kappa \mathrm{B}$, esta última responsable de la regulación del factor tisular y la interleuquina $6\left({ }^{58}\right)$.

Niveles elevados de LDL en sangre representan un factor de riesgo para el desarrollo de la aterosclerosis. Esta lipoproteína permite la adhesión de macrófagos al endotelio vascular. Pero, en realidad, la LDL oxidada (oxLDL) es la que representa un factor muy importante en la inducción de la aterosclerosis, ya que induce la mitosis de los macrófagos, promueve la acumulación de células espumosas, es citotóxica para las células endoteliales e inhibe la vasodilatación que es inducida por el óxido nítrico $\left({ }^{59}\right)$. Esta LDL es oxidada por las células que constituyen la placa aterosclerótica. Un hecho interesante es que uno de los agentes que puede inducir que las células de la placa aterosclerótica conviertan LDL en oxLDL es la C. pneumoniae, evidenciado por la producción de oxLDL en el cultivo de $C$. pneumoniae enriquecido con LDL $\left({ }^{60}\right)$, a través de un mecanismo independiente de iones superóxido, vía que usan los monocitos para oxidar LDLs. Este hallazgo proporciona mayor evidencia de que este organismo puede iniciar, por sí mismo, el proceso de aterosclerosis o acelerar su progresión.

Con relación a otros lípidos, se ha visto que los sujetos con infección crónica por $C$. pneumoniae presentan valores más elevados de triglicéridos y colesterol total, y valores más bajos de lipoproteínas de alta densidad, que los sujetos con anticuerpos negativos $\left({ }^{61,62}\right)$, lo que podría condicionar un alto riesgo para el desarrollo de aterosclerosis. Asimismo, parece ser que el estado hipercolesterolémico aceleraría el proceso aterogénico inducido por la $C$. pneumoniae $\left({ }^{63}\right)$. 
Las proteínas de choque por calor (HSP) se incrementan frente a diversos estímulos; uno de ellos es la inflamación. Mediante el método de inmunohistoquímica se ha detectado HSP 60 de Chlamydia y HSP 60 humana en placas ateromatosas de arterias carótidas humanas. Además, se ha observado experimentalmente en macrófagos de ratón que tanto la HSP 60 de Chlamydia como la HSP 60 humana inducen factor de necrosis tumoral $\alpha$ (citoquina proinflamatoria) -que podría explicar el efecto aterogénico de la $\mathrm{C}$. pneumoniae- y, además, metaloproteinasa, enzima degradante de tejido conectivo, que degradaría la placa fibrosa aterosclerótica, contribuyendo a la desestabilización de la placa y la ocurrencia de un evento coronario agudo $\left(^{6}\right)$. Sasu y col., de la Universidad de Wisconsin, han publicado un interesante trabajo en el que demuestran la capacidad de la $C$. pneumoniae, como cuerpo elemental y como fracción antigénica HSP 60, para inducir la proliferación de células musculares lisas de los vasos, a través de la activación de la proteína quinasa mitógeno-activada p44/p42 $\left({ }^{64}\right)$. Es probable que la mayoría de los efectos de la $C$. pneumoniae en la patogenia de la aterosclerosis sean inducidos por el componente antigénico HSP 60 (Figura 1). La activación de la proteína quinasa mitógeno-activada bajo las isoformas p42 y p44 frente a la exposición de $C$. pneumoniae ha sido previamente demostrada en células endoteliales de la vena umbilical y de la aorta humanas $\left({ }^{65}\right)$, pero no se conoce aún la vía a través de la cual ocurre esta activación, aunque el receptor toll-like-4 está implicado $\left({ }^{64}\right)$. Hasta la fecha, se ha propuesto al mediador MyD88 como una posible vía de señal para la inducción de la respuesta inflamatoria por parte del HSP 60 de la Chlamydia sobre las células endoteliales de la pared de las arterias $\left({ }^{66}\right)$.

Un grupo de investigación alemán no encontró asociación entre los anticuerpos anti-HSP 60 de Chlamydia y enfermedad cardiaca coronaria $\left({ }^{67}\right)$. Sin embargo, el método usado fue ELISA, el cual no ha sido aprobado para el diagnóstico de $C$. pneumoniae, según la CDC. Contrariamente, investigadores italianos liderados por el Dr. Cassone, encontraron títulos elevados de anticuerpos IgG contra el HSP 60 de Chlamydia en más de $99 \%$ de 219 pacientes con enfermedad coronaria, en contraste con la ausencia de anticuerpos en 100 sujetos control pareados por edad y sexo $\left({ }^{68}\right)$. Aunque estos resultados son muy convincentes, fueron obtenidos también usando el método de ELISA y, cuando hicieron la correlación con el método de microinmunofluorescencia indirecta, hubo una pobre correlación. A pesar de esto, con este último método, $47 \%$ de los pacientes con enfermedad coronaria tuvo títulos mayores de 1:128 versus $0 \%$ en el grupo control $\left({ }^{68}\right)$. Cabe destacar que se ha reportado reacciones cruzadas entre el HSP 60 humano y de Chlamydia con la proteina GroEL de la $E$. coli y algunas proteínas de picornavirus $\left({ }^{12}\right)$, por lo que los resultados deben ser tomados con cautela.

Un punto importante a considerar es que, si se piensa que la $C$. pneumoniae está implicada activamente en el proceso de aterosclerosis, entonces debe existir una correlación entre el grado de severidad de la aterosclerosis y el porcentaje de positividad para C. pneumoniae. Erickson y col. $\left({ }^{69}\right)$, demostraron una mayor positividad para $C$. pneumoniae en lesiones ateroscleróticas severas que en las lesiones leves, tanto por inmunohistoquímica ( $80 \%$ de positividad en lesiones severas versus $38 \%$ en lesiones leves), como por inmunofluorescencia directa $(86 \%$ versus $6 \%$, respectivamente). Aunque en este último método usaron kits específicos para género. Nosotros también hemos encontrado correlación entre el grado de severidad de aterosclerosis y el porcentaje de especímenes positivos a $C$. pneumoniae. Esta bacteria fue encontrada en $21 \%$ de los casos de aterosclerosis leve, en $50 \%$ de las lesiones moderadas y en $100 \%$ de casos de aterosclerosis severa $\left({ }^{70}\right)$.

Uno de los mecanismos patogénicos de la $C$. pneumoniae en la enfermedad cardiaca coronaria sería la desestabilización de la placa ateromatosa (fisura o ruptura) y la inducción de un proceso inflamatorio crónico $\left({ }^{15,71}\right)$. Recientemente, hemos demostrado la presencia de $C$. pneumoniae en sujetos fallecidos por IMA, específicamente 


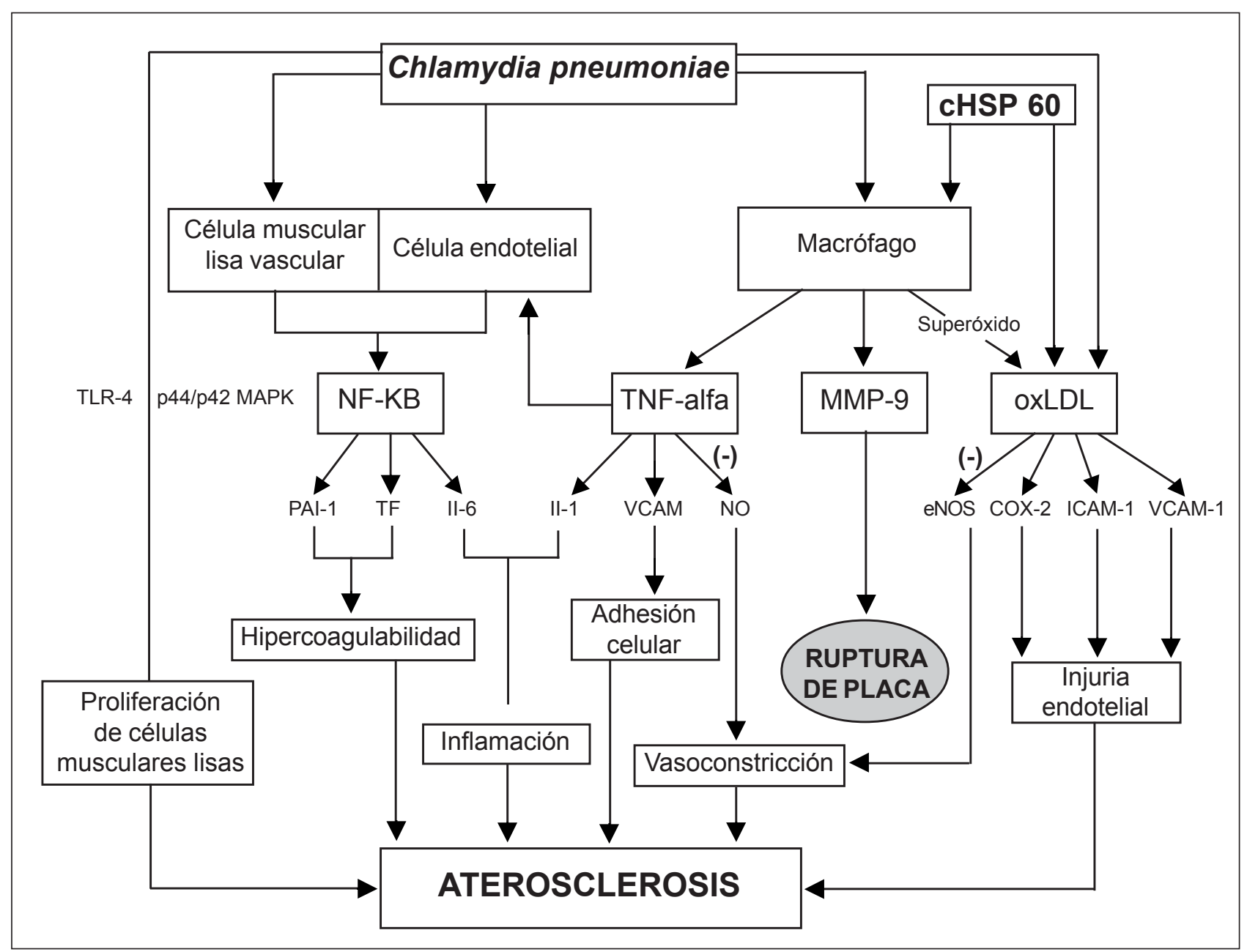

Figura 1.- Esquema hipotético del rol de la Chlamydia pneumoniae en la patogenia de la aterosclerosis. cHSP $60=$ proteína de choque por calor 60 de Chlamydia NF-kappaB = factor nuclear-kappaB; MMP-9= metaloproteinasa de matriz-9; TF = factor tisular; oxLDL = lipoproteína de baja densidad oxidada; TLR = receptor toll-like; MAPK = proteinquinasa mitógeno-activada.

dentro de la lesión aterosclerótica de las arterias coronarias implicadas en el infarto $\left({ }^{70}\right)$, siendo la positividad para $C$. pneumoniae tres veces mayor en los sujetos fallecidos por IMA que en los sujetos fallecidos por causa no cardiaca. Otros investigadores, usando también inmunohistoquímica, han encontrado $84 \%$ de positividad en lesiones ateroscleróticas asociadas a angina inestable o IMA, comparado con $30 \%$ de los casos con angina estable $\left({ }^{72}\right)$.

Para poder atribuir causalidad a un agente infeccioso, la enfermedad tiene que ser reproduci- da en el huésped sano cuando se le inocula una cantidad determinada de este agente infeccioso. Precisamente, se ha logrado inducir aterosclerosis en animales de experimentación, al inocularles cepas de $C$. pneumoniae. Investigadores de la Universidad de Manitoba-Canadá han logrado inducir aterosclerosis en ratones carentes del gen del receptor para LDL, después de 9 meses de inoculación intranasal mensual de $C$. pneumoniae; sin embargo, el efecto aterosclerótico fue significativo sólo en condiciones de hipercolesterolemia ( $\left.{ }^{63}\right)$. En otro experimento, se ha podido observar la aparición de estrías grasas 
y engrosamientos en forma de lunares en la pared del arco aórtico y la aorta torácica en conejos neocelandeses, tan sólo después de 2 semanas de inoculación intranasal de $C$. pneumoniae $\left.{ }^{(39}\right)$, mientras que los controles permanecieron negativos. La aceleración en la progresión de aterosclerosis en ratones deficientes de apolipoproteína-E ha sido demostrado por el grupo liderado por el Dr. Kuo, encontrándose un aumento en el área de las lesiones ateroscleróticas a nivel de la curvatura menor del arco aórtico, $14,3 \pm 11,9 \mu \mathrm{m} 2 \times 105$ en los ratones infectados con C. pneumoniae, comparado con $8,9 \pm 8,8$ $\mu \mathrm{m} 2 \times 105$ en el grupo control $\left({ }^{73}\right)$.

No sólo la $C$. pneumoniae ha sido implicada en la etiopatogenia de la aterosclerosis. Bacterias como el Helicobacter pylori y virus como el Coxsackie B, el citomegalovirus y el virus del herpes simple también han sido involucrados $\left({ }^{23,74-77}\right)$, aunque algunos autores han encontrado sólo asociación con la $C$. pneumoniae y no con otros agentes infecciosos $\left({ }^{40,78}\right)$.

\section{Chlamydia pneumoniae e infarto miocárdico agudo}

En 1988, Saikku y col. demostraron la presencia de IgA e IgG anti-C. pneumoniae en pacientes con infarto miocárdico agudo (IMA) y en pacientes con enfermedad cardiaca coronaria $\left({ }^{79}\right)$; aunque en 1971, Sutton y col. ya habían encontrado una asociación entre Chlamydia y enfermedades cardiacas $\left({ }^{80}\right)$, pero sin utilizar antígenos específicos de la especie pneumoniae.

Posteriormente, en otros trabajos publicados se ha encontrado una importante correlación serológica entre la $C$. pneumoniae y enfermedades cardiacas coronarias $\left({ }^{33,81-83}\right)$. En un estudio prospectivo se encontró una asociación entre títulos de anticuerpos IgA (marcador de infección crónica, aunque no validado para el diagnóstico) contra $C$. pneumoniae y el riesgo de muerte por enfermedades cardiacas isquémicas $\left.{ }^{84}\right)$; sin embargo, esta asociación parece no existir con los anticuerpos IgG $\left({ }^{84-86}\right)$.
Se sabe que los niveles elevados de la proteína $\mathrm{C}$ reactiva se correlacionan con muerte súbita cardiaca e IMA, probablemente porque esta proteína induce la activación del factor tisular, implicado en el inicio del proceso de coagulación $\left.{ }^{87}\right)$. Al respecto, se ha encontrado una fuerte correlación entre esta proteína y la presencia de infección crónica por $C$. pneumoniae en pacientes con angina inestable $\left({ }^{88}\right)$ y en sujetos con factores de riesgo cardiovascular $\left.{ }^{89}\right)$. Nosotros hemos demostrado, mediante inmunohistoquímica, la presencia de $C$. pneumoniae en las lesiones ateroscleróticas de sujetos fallecidos por IMA, con una positividad de $69,2 \%$ en los casos de infarto miocárdico, comparado con $22,7 \%$ en el grupo control, siendo 4,84 veces más frecuente encontrar $C$. pneumoniae en los sujetos que fallecieron por infarto que en los controles $\left({ }^{70}\right)$.

Una explicación inmunológica a la asociación existente entre Chlamydia y enfermedades cardiovasculares sería el mimetismo entre proteínas específicas del miocardio (cadenas pesadas de $\alpha$ miosina específicas del músculo cardiaco) y las proteínas mayores de membrana externa de la $C$. pneumoniae, $C$. psittaci y $C$. trachomatis $\left({ }^{90}\right)$. Este hallazgo sugiere que la C. pneumoniae estaría relacionada con el infarto miocárdico agudo, no sólo por tener un efecto aterogénico sino que también podría actuar directamente sobre el miocardio y causar infarto en un corazón con arterias coronarias normales. Sin embargo, se necesita mayores estudios con relación a este punto.

\section{Chlamydia pneumoniae $\mathbf{y}$ enfermedad cerebrovascular}

Se ha propuesto una teoría infecciosa para explicar la ocurrencia de isquemia cerebral aguda $\left({ }^{19,91-94}\right)$, así como la existente para las enfermedades cardiovasculares. No pocos investigadores han encontrado una asociación entre $C$. pneumoniae y enfermedades cerebrovasculares. En pacientes del Hospital de Birmingham, con diagnóstico de infarto cerebral agudo y de isquemia cerebral transitoria, encontrando títulos de 
anticuerpos anti-C. pneumoniae marcadores de infección aguda en 14,3\%; asimismo, títulos que sugieren infección crónica o previa en 33,9\% ( $\left.{ }^{33}\right)$. Wimmer y col. encontraron títulos elevados de IgA en sujetos con enfermedad cerebrovascular en $46,6 \%$ y $23,1 \%$ en el grupo control, concluyendo que la infección crónica por $C$. pneumoniae está asociada con un riesgo incrementado para la aparición de infarto cerebral o isquemia transitoria $\left({ }^{95}\right)$. En otros estudios también se ha encontrado asociación entre $C$. pneumoniae y enfermedades cerebrovascu-lares $\left({ }^{96,97}\right)$.

Además de existir niveles de anticuerpos contra $C$. pneumoniae más elevados en los pacientes con enfermedades cerebrovasculares de tipo isquémico en comparación con la población control, parece ser que los títulos se correlacionarían con la severidad de la isquemia $\left({ }^{98}\right)$.

\section{¿Antibioticoterapia para la aterosclerosis?}

Desde hace muchos años se ha empleado diversos antibióticos para el tratamiento de la infección respiratoria por C. pneumoniae. Sin embargo, el uso de antibióticos para erradicar esta bacteria de las zonas ateroscleróticas o prevenir enfermedades cardiovasculares todavía es materia de discusión. En general, se ha usado drogas más potentes en estos casos, tales como azitromicina, claritromicina $\left({ }^{99}\right)$ y roxitromicina $\left.{ }^{100}\right)$, aunque se está ensayando también con quinolonas $\left({ }^{99,101}\right)$. No obstante, los estudios que se realizan in vitro no necesariamente reflejan la actividad antimicrobiana en la práctica clínica. Uno de los temas de investigación que ha despertado el interés de muchos investigadores es el relacionado al tratamiento antibiótico de las enfermedades cardiacas coronarias. Se ha realizado estudios prospectivos para determinar la eficacia del tratamiento antibiótico contra $C$. pneumoniae, para prevenir eventos coronarios en pacientes con infarto previo. Uno de ellos encontró una disminución del título de anticuerpos en el grupo de pacientes con infarto previo, después del tratamiento con azitromicina $\left({ }^{102}\right)$. Asi- mismo, en el estudio piloto ROXIS, llevado a cabo en la Unidad Coronaria de Argentina, en el que se usó roxitromicina en pacientes con angina inestable o infarto miocárdico no-Q, se encontró una menor incidencia de eventos coronarios después de 6 meses de seguimiento $\left({ }^{103}\right)$. También se ha ensayado el uso de tetraciclinas y quinolonas con el propósito de disminuir el riesgo de presentar IMA, encontrándose una asociación positiva con estos antibióticos, no así con las cefalosporinas, sulfonamidas ni la eritromicina ${ }^{(104)}$, las cuales tienen una eficacia baja, in vivo, sobre la Chlamydia. Por otro lado, experimentalmente también se ha podido evidenciar la prevención del estrechamiento de la arteria aorta con el uso de azitromicina, en conejos inoculados con cepas de $C$. pneumoniae y dieta rica en colesterol $\left({ }^{105,106}\right)$. En un estudio realizado en ratones deficientes de apolipoproteína-E, al inocularles cepas de $C$. pneumoniae se provocó un incremento en el tamaño de la lesión aterosclerótica aórtica; sin embargo, el uso de azitromicina no mejoró el efecto aterogénico de esta bacteria $\left({ }^{107}\right)$. Además, las dosis usadas habitualmente de tetraciclina, doxiciclina o eritromicina en patologías respiratorias asociadas a $C$. pneumoniae parecen no estar asociadas a una reducción en el riesgo de presentar infarto en el futuro $\left({ }^{108}\right)$.

Entre febrero y marzo de 1999, en los Estados Unidos de Norteamérica, $4 \%$ de los médicos entrevistados ha usado o sugerido el uso de antibióticos para el tratamiento de enfermedades cardiovasculares $\left({ }^{109}\right)$. Los antibióticos ayudarían a disminuir o prevenir futuros eventos coronarios, debido a que, al usar tratamiento específico contra la $C$. pneumoniae, se podría eliminar la bacteria del huésped, cortando así la influencia de este agente en las enfermedades cardiacas. Actualmente se está desarrollando estudios aleatorios, controlados, para determinar el rol del uso de antibióticos en las enfermedades cardiacas coronarias $\left({ }^{12}\right)$. Probablemente, con los resultados de estos estudios de gran escala podremos esclarecer si es que se justifica el uso de antibióticos en estas enfermedades e, indirectamente, si es que la $C$. pneumoniae cumple un rol importante en el proceso de aterogénesis. 
En conclusión, numerosos estudios realizados en diferentes países establecen una asociación entre $C$. pneumoniae y aterosclerosis. Si existe una relación causal aún es incierto. Se está proponiendo algunos mecanismos patogénicos para explicar cómo la $C$. pneumoniae induciría o favorecería la aterosclerosis; sin embargo, las investigaciones llevadas a cabo hasta la fecha representan una fracción del conocimiento que se espera alcanzar en el futuro, para una comprensión más precisa del proceso de la aterosclerosis y consecuentemente para su prevención y tratamiento. La relación de la $C$. pneumoniae con las enfermedades cardiovasculares y cerebrovasculares nos ponen en una disyuntiva: si es justificado usar antibióticos para su tratamiento. Posteriores estudios prospectivos y experimentales nos ayudarán a dilucidar si es que la $C$. pneumoniae constituye el agente causal de la aterosclerosis o tal vez es un agente que acelera el proceso de aterogénesis, con lo cual se estaría justificando el uso de antibióticos en las enfermedades de origen aterosclerótico.

\section{AGRADECIMIENTO}

Al Dr. Ronald Sotelo por su valiosa colaboración en las críticas del manuscrito.

\section{BIBLIOGRAFÍA}

1. Grayston JT, Kuo CC, Wang SP, Altman J. A new Chlamydia psittaci strain, TWAR, isolated in acute respiratory tract infections. N Engl J Med 1986; 315: 161-8.

2. Saikku P, Wang SP, Kleemola M, Brander E, Rusanen E, Grayston JT. An epidemic of mild pneumonia due to an unusual strain of Chlamydia psittaci. J Infect Dis 1985; 151: 832-9.

3. Grayston JT, Campbell LA, Kuo CC, Mordhorst CH, Saikku P, Thom DH, Wang SP. A new respiratory tract pathogen: Chlamydia pneumoniae strain TWAR. J Infect Dis 1990; 161: 618-25.

4. Kuo CC, Chen HH, Wang SP, Grayston JT. Identification of a new group of Chlamydia psittaci strains called TWAR. J Clin Microbiol 1986; 24: 1034-7.

5. Meijer A, Kwakkel GJ, de Vries A, Schouls LM, Ossewaarde JM. Species identification of Chlamydia isolates by analyzing restriction fragment length polymorphism of the $16 \mathrm{~S}-23 \mathrm{~S}$ rRNA spacer region. J Clin Microbiol 1997; 35: 1179-83.
6. Kol A, Sukhova GK, Lichtman AH, Libby P. Chlamydial heat shock protein 60 localizes in human atheroma and regulates macrophage tumor necrosis factor-alpha and matrix metalloproteinase expression. Circulation 1998; 98: 300-7.

7. Peeling RW, Brunham RC. Chlamydiae as pathogens: new species and new issues. Emerg Infect Dis 1996; 2: 307-19.

8. Schachter J, Dawson CR. Psittacosis-lymphogranuloma venereum agents/tric agents. En: Lennette EH, Schmidt NJ, editores. Diagnostic procedures for viral, rickettsial and chlamydial infections. 5ta. ed. American Public Health Association Inc.; 1979: 1021-59.

9. Kuo CC, Gown AM, Benditt EP, Grayston JT. Detection of Chlamydia pneumoniae in aortic lesions of atherosclerosis by immunocytochemical stain. Arterioscler Thromb 1993; 13: 1501-4.

10. Campbell LA, Perez M, Hamilton DJ, Kuo CC, Grayston JT. Detection of Chlamydia pneumoniae by polymerase chain reaction. J Clin Microbiol 1992; 30: 434-9.

11. Kuo CC, Shor A, Campbell LA, Fukushi H, Patton DL, Grayston JT. Demonstration of Chlamydia pneumoniae in atherosclerotic lesions of coronary arteries. J Infect Dis 1993; 167: 841-9.

12. Boman J, Hammerschlag MR. Chlamydia pneumoniae and atherosclerosis: critical assessment of diagnostic methods and relevance to treatment studies. Clin Microbiol Rev 2002; 15: $1-20$.

13. Segal BL. Distribución y relación de la aterosclerosis con la enfermedad cardiaca coronaria. En: Likoff W, Moyer $\mathrm{JH}$, editores. La cardiopatía coronaria. Barcelona: Editorial Científico-Médica; 1967: 163-9.

14. Arbustini E, Morbini P, Dal Bello B, Prati F, Specchia G. From plaque biology to clinical setting. Am Heart J 1999; 138: S55-S60.

15. Muhlestein JB. The link between Chlamydia pneumoniae and atherosclerosis. Infect Med 1997; 14: 380-2.

16. Campbell LA, Kuo CC, Grayston JT. Chlamydia pneumoniae and cardiovascular disease. Emerg Infect Dis 1998; 4: 571-6.

17. Orfila JJ. Seroepidemiological evidence for an association between Chlamydia pneumoniae and atherosclerosis. Atherosclerosis 1998; 140: S11-S15.

18. Taylor-Robinson D. Chlamydia pneumoniae in vascular tissue. Atherosclerosis 1998; 140: S21-S24.

19. Wong YK, Gallagher PJ, Ward ME. Chlamydia pneumoniae and atherosclerosis. Heart 1999; 81: 232-8.

20. Binder S, Levitt AM, Hughes JM. Preventing emerging infectious diseases as we enter the 21st century: CDC's strategy. Public Health Reports 1999; 114: 130-4.

21. Grayston JT. Does Chlamydia pneumoniae cause atherosclerosis? Arch Surg 1999; 134: 930-4.

22. Sullivan JL, Weinberg ED. Iron and the role of Chlamydia pneumoniae in heart disease. Emerg Infect Dis 1999; 5: 724-6.

23. Gupta S, Camm AJ. Chlamydia pneumoniae and coronary heart disease. BMJ 1997; 314: 1778-9.

24. Muhlestein JB, Hammond EH, Carlquist JF, Radicke E, Thomson MJ, Karagounis LA, et al. Increased 
incidence of Chlamydia species within the coronary arteries of patients with symptomatic atherosclerotic versus other forms of cardiovascular disease. J Am Coll Cardiol 1996; 27: 1555-61.

25. Ramírez JA et al. Isolation of Chlamydia pneumoniae from the coronary artery of a patient with coronary atherosclerosis. The Chlamydia pneumoniae/Atherosclerosis Study Group. Ann Intern Med 1996; 125: 979-82.

26. Grayston JT, Campbell LA. The role of Chlamydia pneumoniae in atherosclerosis. Clin Infect Dis 1999; 28: 993-4.

27. Thomas M, Wong Y, Thomas D, Ajaz D, Tsang V, Gallagher PJ, et al. Relation between direct detection of Chlamydia pneumoniae DNA in human coronary arteries at postmortem examination and histological severity (stary grading) of associated atherosclerotic plaque. Circulation 1999; 99: 2733-6.

28. Ramos PM, Ortega F, Samaniego J, Díaz R. Microscopía electrónica, Chlamydia pneumoniae y aterosclerosis. Medicina Clínica 1999; 112: 277-8.

29. Grayston JT, Kuo CC, Coulson AS, Campbell LA, Lawrence RD, Lee MJ, et al. Chlamydia pneumoniae (TWAR) in atherosclerosis of the carotid artery. Circulation 1995; 92: 3397-400.

30. Wissler RW. Significance of Chlamydia pneumoniae (TWAR) in atherosclerotic lesions. Circulation 1995; 92: 3376.

31. Thom DH, Grayston JT, Siscovick DS, Wang SP, Weiss NS, Daling JR. Association of prior infection with Chlamydia pneumoniae and angiographically demonstrated coronary artery disease. JAMA 1992; 268: 68-72.

32. Puolakkainen M, Kuo CC, Shor A, Wang SP, Grayston JT, Campbell LA. Serological response to Chlamydia pneumoniae in adults with coronary arterial fatty streaks and fibrolipid plaques. J Clin Microbiol 1993; 31: 2212-4.

33. Cook PJ, Honeybourne D, Lip GYH, Beevers DG, Wise R. Chlamydia pneumoniae and acute arterial thrombotic disease. Circulation 1995; 92: 3148-9.

34. Miyashita N, Toyota E, Sawayama T, Matsumoto A, Mikami Y, Kawai N, et al. Association of chronic infection of Chlamydia pneumoniae and coronary heart disease in the japanese. Intern Med 1998; 37: 913-6.

35. Melnick SL, Shahar E, Folsom AR, Grayston JT, Sorlie PD, Wang SP, et al. Past infection by Chlamydia pneumoniae strain TWAR and asymptomatic carotid atherosclerosis. Am J Med 1993; 95: 499-504.

36. Stenvinkel P, Heimbürger $\mathbf{O}$, Jogestrand T, Kärnell A, Samuelsson A. Does persistent infection with Chlamydia pneumoniae increase the risk of atherosclerosis in chronic renal failure? Kidney Int 1999; 55: 2531-2.

37. Jackson LA, Campbell LA, Schmidt RA, Kuo CC, Cappuccio AL, Lee MJ, et al. Specificity of detection of Chlamydia pneumoniae in cardiovascular atheroma. Am J Pathol 1997; 150: 1785-90.

38. Marrie TJ, Grayston JT, Wang SP, Kuo CC. Pneumonia associated with the TWAR strain of Chlamydia. Ann Intern Med 1987; 106: 507-11.
39. Laitinen K, Laurila A, Pyhälä L, Leinonen M, Saikku P. Chlamydia pneumoniae infection induces inflammatory changes in the aortas of rabbits. Infect Immun 1997; 65: 4832-5.

40. Bartel C, Maass M, Bein G, Malisius R, Brill N, Bechtel $\mathbf{M}$, et al. Detection of Chlamydia pneumoniae but not cytomegalovirus in occluded saphenous vein coronary artery bypass grafts. Circulation 1999; 99: 879-82.

41. Jantos CA, Nesseler A, Waas W, Baumgärtner W, Tillmanns H, Haberbosch W. Low prevalence of Chlamydia pneumoniae in atherectomy specimens from patients with coronary heart disease. Clin Infect Dis 1999; 28: 988-92.

42. Wong YK, Thomas M, Tsang V, Gallagher PJ, Ward ME. The prevalence of Chlamydia pneumoniae in atherosclerotic and nonatherosclerotic blood vessels of patients attending for redo and first time coronary artery bypass graft surgery. J Am Coll Cardiol 1999; 33: 152-6.

43. Hammerschlag MR. Current knowledge of Chlamydia pneumoniae and atherosclerosis. Eur J Clin Microbiol Infect Dis 1998; 17: 305-8.

44. Maass M, Bartels C, Engel PM, Mamat U, Sievers HH. Endovascular presence of viable Chlamydia pneumoniae is a common phenomenon in coronary artery disease. J Am Coll Cardiol 1998; 31: 827-32.

45. Gurfinkel E. Chlamydia pneumoniae in redo and first-time coronary artery bypass graft surgery. J Am Coll Cardiol 1999; 34: 953-4.

46. Capron L. Peut-on attraper un infarctus? La Recherche 1997; 294: 32-4.

47. Fang JC, Kinlay S, Kundsin R, Ganz P. Chlamydia pneumoniae infection is frequent but not associated with coronary arteriosclerosis in cardiac transplant recipients. Am J Cardiol 1998; 82: 1479-83.

48. Coles KA, Plant AJ, Riley TV, Smith DW, McQuillan BM, Thompson PL. Lack of association between seropositivity to Chlamydia pneumoniae and carotid atherosclerosis. Am J Cardiol 1999; 84: 825-8.

49. Markus HS, Sitzer M, Carrington D, Mendall M, Steinmetz. Chlamydia pneumoniae infection and early asymptomatic carotid atherosclerotic. Circulation 1999; 100: 832-7.

50. Hoffmeister A, Rothenbacher D, Wanner P, Bode G, Persson K, Hombach V, et al. Seropositivity to chlamydial lipopolysaccharide and chlamydia pneumoniae, systemic inflammation and stable coronary artery disease: Negative results of a case-control study. J Am Coll Cardiol 2000; 35: 112-8.

51. Dowell SF, Boman J, Carlone GM, Fields BS, Guarner J, Hammerschlag MR, et al. Standardizing Chlamydia pneumoniae assays: recommendations from the Centers for Disease Control and Prevention (USA), and the Laboratory Centre for Disease Control (Canada). Clin Infect Dis 2001; 33: 492-503.

52. Ross R, Glomset JA. The pathogenesis of atherosclerosis. N Engl J Med 1976; 295: 369-76. 
53. Ross R. The pathogenesis of atherosclerosis: a perspective for the 1990s. Nature 1993; 362: 801-9.

54. Noll G. Pathogenesis of atherosclerosis: a possible relation to infection. Atherosclerosis 1998; 140: S3-S9.

55. Godzik KL, O'Brien ER, Wang SK, Kuo CC. In vitro susceptibility of human vascular wall cells to infection with Chlamydia pneumoniae. J Clin Microbiol 1995; 33: 2411-4.

56. Yamashita K, Ouchi K, Shirai M, Gondo T, Nakazawa T, Ito H. Distribution of Chlamydia pneumoniae infection in the atherosclerotic carotid artery. Stroke 1998; 29: 773-8.

57. Blasi F, Cosentini R, Raccanelli R, Massari FM. A possible association of Chlamydia pneumoniae infection and acute myocardial infarction in patients younger than 65 years of age. Chest 1997; 112: 309-12.

58. Dechend R, Maass M, Gieffers J, Dietz R, Scheidereit C, Leutz A, et al. Chlamydia pneumoniae infection of vascular smooth muscle and endothelial cells activates NFkappaB and induces tissue factor and PAI-1 expression. Circulation 1999; 100: 1369-73.

59. Steinberg D. Low density lipoprotein oxidation and its pathobiological significance. J Biol Chem 1997; 272: 20963-6.

60. Kalayoglu MV, Hoerneman B, LaVerda D, Morrison SG, Morrison RP, Byrne GI. Cellular oxidation of lowdensity lipoprotein by Chlamydia pneumoniae. J Infect Dis 1999; 180: 780-90.

61. Laurila A, Bloigu A, Näyhä S, Hassi J, Leinonen M, Saikku P. Chlamydia pneumoniae antibodies and serum lipids in Finnish men: cross sectional study. BMJ 1997; 314: 1456-7.

62. Laurila A, Bloigu A, Näyhä S, Hassi J, Leinonen M, Saikku P. Chronic Chlamydia pneumoniae infection is associated with a serum lipid profile known to be a risk factor for atherosclerosis. Arterioscler Thromb Vasc Biol 1997; 17: 2910-3.

63. Hu H, Pierce GN, Zhong G. The atherogenic effects of chlamydia are dependent on serum cholesterol and specific to Chlamydia pneumoniae. J Clin Invest 1999; 103: 747-53.

64. Sasu S, LaVerda D, Qureshi N, Golenbock DT, Beasley D. Chlamydia pneumoniae and chlamydial heat shock protein 60 stimulate proliferation of human vascular smooth muscle cells via toll-like receptor 4 and p44/p42 mitogen-activated protein kinase activation. Circ Res 2001; 89: 244-50.

65. Krüll M, Klucken AC, Wuppemann FN, Fuhrmann O, Magerl C, Seybold J, et al. Signal transduction pathways activated in endothelial cells following infection with Chlamydia pneumoniae. J Immunol 1999; 162: 4834-41.

66. Bulut Y, Faure E, Thomas L, Karahashi H, Michelsen KS, Equils O, et al. Chlamydial heat shock protein 60 activates macrophages and endothelial cells through tolllike receptor 4 and MD2 in a MyD88-dependent pathway. J Immunol 2002; 168: 1435-40.

67. Jantos CA, Krombach C, Wuppermann FN, Gardemann A, Bepler S, Asslan H, et al. Antibody response to the 60$\mathrm{kDa}$ heat-shock protein of Chlamydia pneumoniae in patients with coronary artery disease. J Infect Dis 2000; 181: 1700-5.
68. Ciervo A, Visca P, Petrucca A, Biasucci LM, Maseri A, Cassone A. Antibodies to 60-kilodalton heat shock protein and outer membrane protein 2 of Chlamydia pneumoniae in patients with coronary heart disease. Clin Diag Lab Immunol 2002; 9: 66-74

69. Ericson K, Saldeen TGP, Lindquist O, Påhlson C, Mehta JL. Relationship of Chlamydia pneumoniae infection to severity of human coronary atherosclerosis. Circulation 2000; 101: 2568-71.

70. Woolcott OO, Sánchez LM, Ruiz E, Maguiña J, Carrera R, Aronés S, et al. Detección de Chlamydia pneumoniae en lesiones ateroscleróticas asociadas a infarto miocárdico agudo. An Fac Med 2001; 62: 325-31.

71. Gurfinkel E, Bazovich G. Chlamydia pneumoniae: inflammation and instability of the atherosclerosis plaque. Atherosclerosis 1998; 140: S31-S35.

72. Bauriedel G, Welsch U, Likungu JA, Welz A, Lüderitz B. Chlamydia pneumoniae in koronarem plaquegewebe: Vermehrter nachweis bei akutem koronarsyndrom. Dtsch med Wschr 1999; 124: 375-80.

73. Moazed TC, Campbell LA, Rosenfeld ME, Grayston JT, Kuo CC. Chlamydia pneumoniae infection accelerates the progression of atherosclerosis in apolipoprotein E-deficient mice. J Infect Dis 1999; 180: 238-41.

74. Grayston JT. Antibiotic tratment of Chlamydia pneumoniae for secondary prevention of cardiovascular events. Circulation 1998; 97: 1669-70.

75. Gura T. Infection: A cause of artery-clogging plaques? Science 1998; 281: 35-7.

76. Pasceri V, Cammarota G, Patti G, Cuoco L, Gasbarrini A, Grillo RL, et al. Association of virulent Helicobacter pylori strains with ischemic heart disease. Circulation 1998; 97: 1675-9.

77. Kuvin JT, Kimmelstiel CD. Infectious causes of atherosclerosis. Am Heart J 1999; 137: 216-26.

78. Blasi F, Denti F, Erba M, Cosentini R, Raccanelli R, Rinaldi A, et al. Detection of Chlamydia pneumoniae but not Helicobacter pylori in atherosclerotic plaques of aortic aneurysms. J Clin Microbiol 1996; 34: 2766-9.

79. Saikku P, Mattila K, Nieminen MS, Huttunen JK, Leinonen M, Ekman MR, et al. Serological evidence of an association of a novel Chlamydia, TWAR, with chronic coronary heart disease and acute myocardial infarction. Lancet 1988; 2: 983-6.

80. Sutton GC, Demakis JA, Anderson TO, Morrissey RA. Serological evidence of a sporadic outbreak in Illinois of infection by Chlamydia (psittacosis-LGV agent) in patients with primary myocardial disease and respiratory disease. Am Heart J 1971; 81: 597-607.

81. Saikku P, Leinonen M, Tenkanen L, Linnanmäki E, Ekman MR, Manninen V, et al. Chronic Chlamydia pneumoniae infection as a risk factor for coronary heart disease in the Helsinki Heart Study. Ann Intern Med 1992; 116: 273-8.

82. Mazzoli S, Tofani N, Semplici F, et al. Chlamydia pneumoniae antibody response in patients with acute 
myocardial infarction and their follow-up. Am Heart J 1998; 135: $15-20$.

83. Nieto FJ, Folsom AR, Sorlie PD, Grayston JT, Wang SP, Chambless LE. Chlamydia pneumoniae infection and incident coronry heart disease. Am J Epidemiol 1999; 150: 149-56.

84. Strachan DP, Carrington D, Mendall MA, Ballam L, Morris J, Butland BK, et al. Relation of Chlamydia pneumoniae serology to mortality and incidence of ischaemic heart disease over 13 years in the Caerphilly prospective heart disease study. BMJ 1999; 318: 1035-40.

85. Ridker PM, Hennekens CH, Buring JE, Kundsin R, Shih J. Baseline IgG antibody titers to Chlamydia pneumoniae, Helicobacter pylori, herpes simplex virus, and cytomegalovirus and the risk for cardiovascular disease in women. Ann Intern Med 1999; 131: 573-7.

86. Ridker PM, Kundsin RB, Stampfer MJ, Poulin S, Hennekens CH. Prospective study of Chlamydia pneumoniae IgG seropositivity and risks of future myocardial infarction. Circulation 1999; 99: 1161-4.

87. Abdelmouttaleb I, Danchin N, Ilardo C, Aimone-Gastin I, Angioï M, Lozniewski A, et al. C-reactive protein and coronary artery disease: Additional evidence of the implication of an inflammatory process in acute coronary syndromes. Am Heart J 1999; 137: 346-51.

88. Biasucci LM, Liuzzo G, Grillo RL, Caligiuri G, Rebuzzi AG, Buffon A, et al. Elevated levels of C-reactive protein at discharge in patients with unstable angina predict recurrent instability. Circulation 1999; 99: 855-60.

89. Mendall MA, Patel P, Ballam L, Strachan D, Northfield TC. $C$ reactive protein and its relation to cardiovascular risk factors: a population based cross sectional study. BMJ 1996; 312: 1061-5.

90. Bachmaier K, Neu N, de la Maza LM, Pal S, Hessel A, Penninger JM. Chlamydia and heart disease linked through antigenic mimicry. Science 1999; 283: 1335-9.

91. Grau AJ, Buggle F, Heindl S, Steichen-Wiehn C, Banerjee T, Maiwald M, et al. Recent infection as a risk factor for cerebrovascular ischemia. Stroke 1995; 26: 373-9.

92. Bova IY, Bornstein NM, Korczyn AD. Acute infection as a risk factor for ischemic stroke. Stroke 1996; 27: 2204-6.

93. Grau AJ, Buggle F, Ziegler C, Schwarz W, Meuser J, Tasman AJ, et al. Association between acute cerebrovascular ischemia and chronic and recurrent infection. Stroke 1997; 28: 1724-9.

94. Grau AJ, Buggle F, Becher H. Zimmermann E, Spiel M, Fent T, et al. Recent bacterial and viral infection is a risk factor for cerebrovascular ischemia. Neurology 1998; 50: 196-203.

95. Wimmer MLJ, Sandmann-Strupp R, Saikku P, Haberl RL. Association of Chlamydial infection with cerebrovascular disease. Stroke 1996; 27: 2207-10.
96. Cook PJ, Honeybourne D, Lip GYH, Beevers G, Wise $\mathbf{R}$, Davies P. Chlamydia pneumoniae antibody titers are significantly associated with acute stroke and transient cerebral ischemia. The West Birmingham Stroke Project. Stroke 1998; 29: 404-10.

97. Fagerberg B, Gnarpe J, Gnarpe H, Agewall S, Wikstrand J. Chlamydia pneumoniae but not Cytomegalovirus antibodies are associated with future risk of stroke and cardiovascular disease. A prospective study in middle-aged to elderly men with treated hypertension. Stroke 1999; 30: 299-305.

98. Grau AJ, Buggle F, Steichen-Wiehn C, Heindl S, Banerjee T, Seitz R, et al. Clinical and biochemical analysis in infection-associated stroke. Stroke 1995; 26: 1520-6.

99. Hammerschlag MR. Activity of quinolones against Chlamydia pneumoniae. Drugs 1999:58: Suppl 2: 78-81.

100.Schussheim AE, Fuster V. Antibiotics for myocardial infarction? Drugs 1999; 57: 283-91.

101.Roblin PM, Reznik T, Kutlin A, Hammerschlag MR. In vitro activities of gemifloxacin (SB 265805, LB20304) against recent clinical isolates of Chlamydia pneumoniae. Antimicrob Agents Chemother 1999; 43: 2806-7.

102.Gupta S, Leatham EW, Carrington D, Mendall MA, Kaski JC, Camm AJ. Elevated Chlamydia pneumoniae antibodies, cardiovascular events, and azithromycin in male survivors of myocardial infarction. Circulation 1997; 96: 404-7.

103. Gurfinkel E, Bozovich G, Daroca A, Beck E, Mautner B. Randomized trial of roxithromycin in non-Q-wave coronary syndromes: ROXIS pilot study. Lancet 1997; 350: 404-7.

104. Meier CR, Derby LE, Jick SS, Vasilakis C, Jick H. Antibiotics and risk of subsequent first-time acute myocardial infarction. JAMA 1999; 281: 427-31.

105. Muhlestein JB, Anderson JL, Hammond EH, Zhao L, Trehan S, Schwobe EP, et al. Infection with Chlamydia pneumoniae accelerates the development of atherosclerosis and treatment with azithromycin prevents it in a rabbit model. Circulation 1998; 97: 633-6.

106. Bauriedel G, Hutter R, Lüderitz B. Azithromycin, Chlamydia pneumoniae, and intimal thickening. Circulation 1998; 98: 2357.

107. Rothstein NM, Quinn TC, Madico G, Gaydos CA, Lowenstein CJ. Effect of azithromycin on murine arteriosclerosis exacerbated by Chlamydia pneumoniae. J Infect Dis 2001; 183: 232-8.

108.Giménez-Sánchez F, Butler JC, Jernigan DB, Strausbaugh LJ, Slemp CC, Perilla MJ, et al. Treating cardiovascular disease with antimicrobial agents: A survey of knowledge, attitudes, and practices among physicians in the United States. Clin Infect Dis 2001; 33: 171-6.

109.Jackson LA, Smith NL, Heckbert SR, Grayston JT, Siscovick DS, Psaty BM. Lack of association between first myocardial infarction and past use of erythromycin, tetracycline, or doxycycline. Emerg Infect Dis 1999; 5: 281-4. 